\title{
Review Article \\ Cancer Stem Cells and Their Microenvironment: Biology and Therapeutic Implications
}

\author{
Eunice Yuen-Ting Lau, ${ }^{1,2}$ Nicole Pui-Yu Ho, ${ }^{1,2}$ and Terence Kin-Wah Lee \\ ${ }^{1}$ Department of Applied Biology and Chemical Technology, The Hong Kong Polytechnic University, Kowloon, Hong Kong \\ ${ }^{2}$ State Key Laboratory for Liver Research, The University of Hong Kong, Pokfulam, Hong Kong \\ Correspondence should be addressed to Terence Kin-Wah Lee; terence.kw.lee@polyu.edu.hk
}

Received 9 January 2017; Accepted 9 February 2017; Published 26 February 2017

Academic Editor: Xiaojiang Cui

Copyright (c) 2017 Eunice Yuen-Ting Lau et al. This is an open access article distributed under the Creative Commons Attribution License, which permits unrestricted use, distribution, and reproduction in any medium, provided the original work is properly cited.

Tumor consists of heterogeneous cancer cells including cancer stem cells (CSCs) that can terminally differentiate into tumor bulk. Normal stem cells in normal organs regulate self-renewal within a stem cell niche. Likewise, accumulating evidence has also suggested that CSCs are maintained extrinsically within the tumor microenvironment, which includes both cellular and physical factors. Here, we review the significance of stromal cells, immune cells, extracellular matrix, tumor stiffness, and hypoxia in regulation of CSC plasticity and therapeutic resistance. With a better understanding of how CSC interacts with its niche, we are able to identify potential therapeutic targets for the development of more effective treatments against cancer.

\section{Introduction}

Cancer exists as a heterogeneous population, with different cancer cells showing distinct phenotypic and functional properties, leading to the limitation of therapeutic efficacy and treatment outcomes. In fact, the discovery of the "Cancer Stem Cell (CSC)/Tumor-Initiating Cell (T-IC)" theory provides an alternative explanation for the failure of existing therapies. Although the idea of CSCs was proposed over a decade ago, the existence of CSCs has been identified in various types of cancer by taking the advantage of available cell surface markers in the last 10 years. In this model, cancer cells are organized in a hierarchy with cancer stem cells (CSCs)/Tumor-Initiating Cell (T-IC) located at the apex [1]. The new concept of CSCs is based on the idea that stem cells are present in cancer tissue, like in normal tissues, and are part of the hierarchy of cells. In other words, just as there are normal stem cells in normal tissues, CSCs are found in tumor tissues. Although the origin of CSCs remains controversial, there is increasing evidence to support that CSCs arise by either mutation from normal stem/progenitor cells or deregulation of genetic programs regulating these cells. These acquired mutations allow normal stem cells to transform from their quiescent and tightly regulated phenotype to constitutively activated ones. This model proposes that CSCs, which share some similar functional properties with normal stem cells, possess the ability to self-renew and initiate tumor formation and generate additional differentiated progenies that compose the heterogeneous tumor bulk. Furthermore, mounting evidence has shown that CSCs are protected by multiple resistance mechanisms, leading to tumor metastasis, therapeutic resistance, and recurrence. Therefore, CSCtargeting therapies represent a promising strategy for the long-term cure of the disease.

And in theory, stem/progenitor cells represent the natural target of tumorigenic mutations since they are possibly the only cells that have the longevity and are endowed with the appropriate capabilities to accumulate the required number of mutations needed to disrupt intrinsic mechanism regulating normal cell proliferation and differentiation $[2$, 3].

In a normal organ, stem cells reside in a "stem cell niche," a specific microenvironment that plays a key role in regulating stem cell maintenance and self-renewal by secreting various paracrine factors or by direct cell-cell 


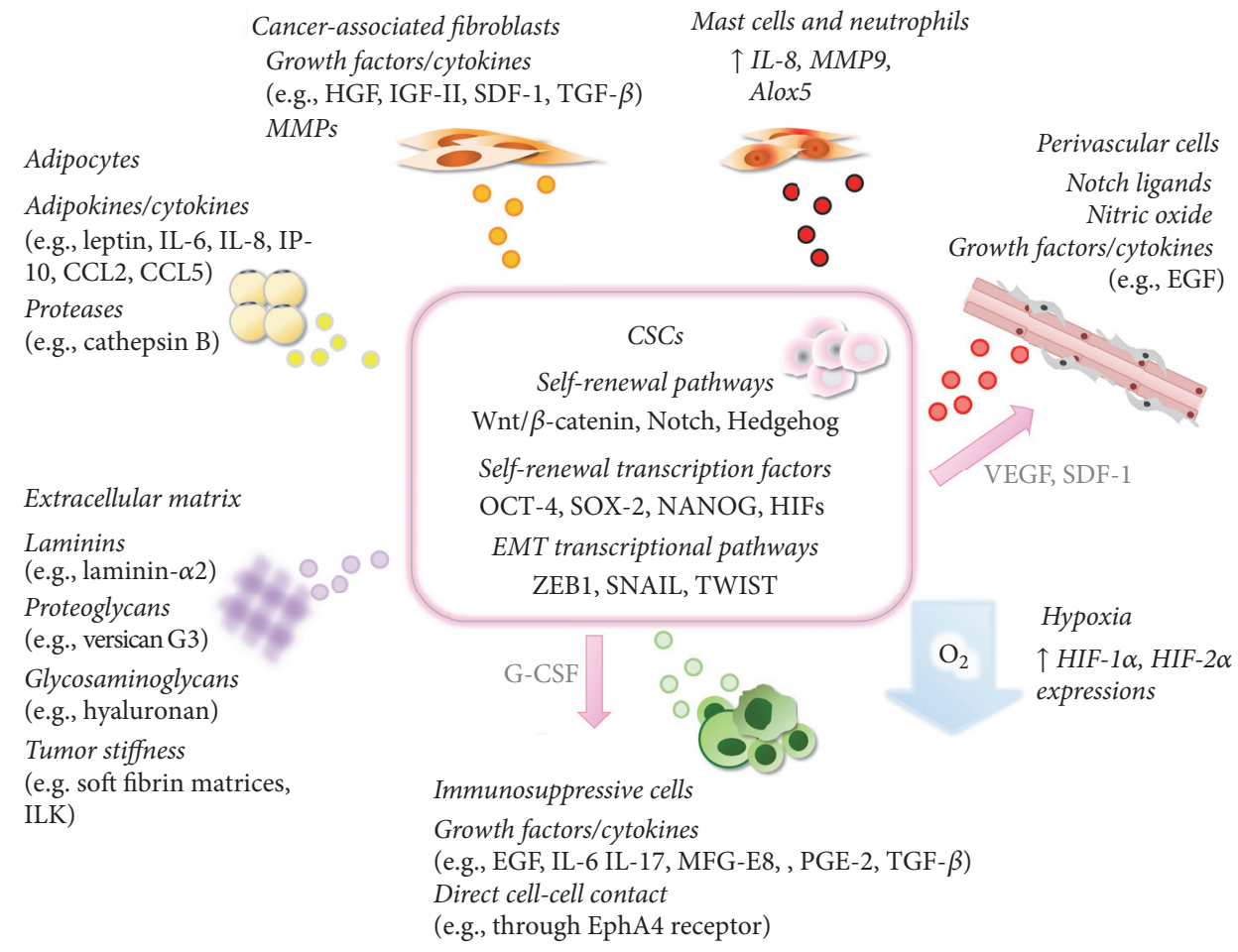

FIGURE 1: The cross talks between CSCs and their niches. CSCs reside in their habitats, which are specific microenvironments within the tumor consisting of CAFs, masts cells, neutrophils, perivascular cells, adipocytes, ECM, and immune-suppressive cells, as well as hypoxia. By providing various paracrine factors or via direct cell-cell contact, these niches play a crucial role in maintaining CSC plasticity by regulating pathways or transcription factors involved in self-renewal or EMT process. Reciprocally, CSCs can actively recruit some of these niche components to create a microenvironment that is favorable for its survival. For example, CSCs can secrete VEGF and SDF-1 to recruit perivascular cells or produce G-CSF to recruit MDSCs into the tumor microenvironment.

contact that interferes with self-renewal and differentiation pathways. A similar concept applies to CSCs in which a cancer-specific "cancer stem cell niche" is also present and interactions with this niche are essential for maintaining the CSC population. Tumor specific microenvironments comprise stromal cells, immune cells, networks of cytokines and growth factors, hypoxic regions, and the extracellular matrix (ECM) (Figure 1). These environmental factors collectively maintain the stemness of CSCs through altering self-renewal pathways, such as the Wnt/ $\beta$-catenin, Notch, and Hedgehog pathways, or by interrupting the master transcriptional regulators that sustain embryonic stem cell self-renewal, such as NANOG, OCT-4, and SOX-2 [2, 3]. Furthermore, extensive evidence has revealed that cancers do not strictly follow the CSC model and the actual CSC model is more complex and flexible. Given a specific environmental stimulus, certain cancer cells exhibit plasticity, enabling these cells to reversibly convert from differentiated to a stem-like state through dedifferentiation processes, such as the epithelial-tomesenchymal transition (EMT) [4]. Considering the essential role of the tumor microenvironment in regulating the CSC phenotype, this review will focus on the recent findings on the molecular mechanisms involved in the cross talk between CSCs and their niches which contributes to maintaining the CSC population.

\section{Stromal Cells}

2.1. Cancer-Associated Fibroblasts. Cancer-associated fibroblasts (CAFs) are the major components of the tumor stroma $[10,11]$. Recent studies have revealed that CAFs are a heterogeneous population, most of which acquire the activated phenotype with increased contractile force, proliferative activity, and enhanced secretion of ECM, proteases, and growth factors. CAFs emerge from multiple origins that widely vary among different cancer types. Several studies have shown that cancer cells could actually secrete signaling molecules, such as basic fibroblast growth factor (bFGF), transforming growth factor beta $(\mathrm{TGF}-\beta)$, platelet-derived growth factor (PDGF), and interleukin- (IL-) 6 to "educate" resting fibroblasts to become CAFs [12-15], and in turn, CAFs promote tumor growth and sustain the stemness property of CSCs in a paracrine manner. Through the secretion of hepatocyte growth factor (HGF), CAFs from colon cancer were demonstrated to support CSC properties through the induction of Wnt/ $\beta$-catenin signaling [16]. More interestingly, the paracrine activation of $\mathrm{Wnt} / \beta$-catenin signaling by CAFs could restore the stem-like features of non-CSCs, thereby expanding the pool of these cells. Using conditioned media from CAFs, we showed that CAFs from liver cancer promote cancer stemness through the noncanonical induction of the 
Notch signaling effector HEY-1 mediated by HGF [17]. A recent study also demonstrated that CAFs in lung cancer induce the expression of the NANOG transcription network through paracrine insulin-like growth factor II (IGF-II)/IGF$1 \mathrm{R}$ signaling [18]. EMT is the process where cancer cells acquire a mesenchymal trait and become more invasive and metastatic. Cancer cells that have undergone EMT typically acquire an increased stemness property because some of the EMT-mediating transcription factors, such as Snail and ZEB1, are essential for self-renewal. Several studies have also shown that the activation of EMT could induce the generation of the CSC population $[19,20]$. In prostate cancer, CAFs can elicit EMT and increase the stemness properties of cancer cells through the secretion of MMPs [13]. Furthermore, CAFs from breast cancer have been reported to promote the EMT of cancer cells via the secretion of stromal-derived factor 1 (SDF-1) and TGF- $\beta 1[21,22]$, providing additional support, suggesting that CAFs play a crucial role in promoting cancer stemness.

2.2. Adipocytes. Obesity is a well-recognized risk factor of several common human malignancies, including breast cancer, colon cancer, and liver cancer [23]. In addition to its epidemic significance, emerging studies have uncovered the functional role of adipose tissues in carcinogenesis and cancer progression, particularly in cancers with adipose tissue constituting a major part of the tumor microenvironment. Adipose tissue primarily comprises adipocytes and a variety of cells that make up the stromal vascular fraction. In addition to its lipid storage function, adipocytes can actively secrete multiple adipokines and cytokines, such as leptin, adiponectin, IL-6, MCP-1, and TNF- $\alpha$, during excessive adiposity [24]. In addition to its role in lipid homeostasis, many of these adipokines and cytokines are proinflammatory, which attract the infiltration of inflammatory cells, particularly macrophages, causing chronic inflammation to promote cancer growth and metastasis. Furthermore, some of these adipocyte-secreted adipokines/cytokines were directly involved in regulating CSCs. In breast cancer, the expression of leptin receptor is highly upregulated in tumor tissue, particularly in the CSC subpopulation, as driven by the selfrenewal associated transcription factors OCT-4 and SOX-2. The secretion of leptin by adipocytes activates the STAT3 signaling in CSCs and induces the expression of OCT-4 and SOX-2, in turn stimulating the expression of leptin receptor, which maintains a self-reinforcing signaling cascade to expand the CSC population and promote tumor growth [25]. Another study showed that the coculture of adipocytes and breast cancer cells stimulates the production of various cytokines that promote cancer stemness through the $\mathrm{Src} / \mathrm{SOX}-2 / \mathrm{miR}-302 \mathrm{~b}$ signaling pathway [26]. In prostate cancer, where obesity is associated with a more aggressive phenotype, adipocytes produce cathepsin B (CTSB) upon coculture with prostate cancer cells to support the selfrenewal of CSCs [27]. Adipocytes from colorectal cancer are also demonstrated to enhance cancer stemness, and their oncogenic function can be impaired by grape seed extract, a well proven agent with anticolorectal cancer activity, through inducing the "browning" of adipocytes [28].
2.3. Perivascular Cells. Angiogenesis is essential for tumor growth and metastasis. With the excessive production of proangiogenic factors by cancer cells, tumors typically develop disorganized and rich blood vessel networks to meet the high demand on oxygen and nutrients required for tumor outgrowth. CSCs promote tumor angiogenesis. For example, in brain, skin, pancreatic, and liver cancer, the $\mathrm{CD}_{133^{+}}$CSC populations produce higher levels of proangiogenic factors, such as vascular endothelial growth factor (VEGF) and SDF-1, recruit more endothelial cells, and stimulate more tube formation compared with their differentiated $\mathrm{CD} 133^{-}$ counterparts [29-31]. Intriguingly, glioblastoma stem cells, which reside in the perivascular niche, undergo differentiation to generate vascular pericytes and endothelial cells to expand tumor vascularization [32,33]. Indeed, a mean of approximately $60 \%$ of endothelial cells in glioblastoma are derived from neoplastic cells [33]. In turn, CSCs reside in close proximity to the perivascular niche, which provides functional support. Strong evidence suggests that vascular endothelial cells play a key role in maintaining CSCs. In the context of glioblastoma, endothelial cells provide Notch ligands to neighboring CSCs, activating Notch signaling and promoting CSCs self-renewal [34]. In another study, perivascular endothelial cells were demonstrated to activate Notch signaling in glioma stem cells through another soluble factor, nitric oxide [35]. A similar observation was also made in colon cancer, suggesting that endothelial cells secrete the Notch ligand Jagged-1 to promote colon CSC phenotype [36]. A recent study on head and neck cancer also highlighted a role for endothelial cells in regulating CSCs, in which endothelial cells were shown to secrete epidermal growth factor (EGF) to induce EMT and promote cancer stemness [37]. Together, these findings reveal an intriguing reciprocal interaction between CSCs and perivascular cells.

2.4. CSCs and Immune Evasion. Tumor immune escape is a fundamental step for tumor development and the major reason for the failure in cancer immunotherapy. Cancer cells evade the infiltration and the cytotoxic function of natural killer (NK) $\mathrm{T}$ cells and $\mathrm{CD}^{+}$cytotoxic $\mathrm{T}$ cells through various strategies, including the active attraction of immunesuppressive cells, production of immune-suppressive factors, and the activation of "immune checkpoints" that induce anergy or apoptosis in T lymphocytes to downmodulate immune functions $[38,39]$. Several studies have revealed that the activation of prosurvival pathways, such as PI3K/AKT, in CSCs not only facilitates escape from conventional chemotherapies but also confers immune evasion [40]. The expression of MHC-I and MHC-II proteins, required for recognition by $\mathrm{T}$ lymphocytes to elicit immune responses, is also downregulated in CSCs [41]. In head and neck cancer, the programmed death-ligand 1 (PD-L1), which binds to the programmed death 1 (PD-1) receptor on $\mathrm{T}$ cells to suppress its function, is selectively expressed on CD $44^{+} \mathrm{CSCs}$ [42]. Furthermore, it has been well documented that CSCs actively recruit immune-suppressive cells into the tumor microenvironment. In addition to functions in modulating immune cells, these tumor-associated immune-suppressive cells, which mainly include tumor-associated macrophages, 
myeloid-derived suppressor cells (MDSCs), T-regulatory (Treg) cells, and NK cells, have been widely demonstrated to support CSCs through multiple pathways.

2.5. Tumor-Associated Macrophages. Macrophages are classified into M1- and M2-polarized subtypes. The M1-subtype secretes inflammatory cytokines and reactive oxygen intermediates and presents antigen to tumor suppressive $\mathrm{T}$ cells. However, the M2-subtypes, which are tumor promoting, induce $\mathrm{T}$ cell anergy, produce extracellular matrix components, repair damaged tissues, and induce angiogenesis [4345]. Although the origins of macrophages in many cancers remain uncertain, most of the macrophages recruited to the tumor microenvironment, known as the TAMs, become the tumor supportive M2 subtype [46]. In glioblastoma, glioma CSCs activate the STAT3 pathway to produce cytokines, which recruit and polarize macrophages to become M2-like [47]. After recruitment, TAMs, in turn, serve as a CSC niche to support CSC growth. For example, in breast cancer, the physical interaction between TAMs and CSCs activates the EphA4 receptor on CSCs and the downstream Src and NF$\kappa \mathrm{B}$ pathways, which promote self-renewal [48]. In a murine model of breast cancer, TAMs are also demonstrated to promote CSC phenotypes in breast cancer cells through the EGFmediated STAT3/SOX-2 cascade, and this cross talk could be abrogated by small molecule inhibitors against EGFR or STAT3 [49]. TGF- $\beta 1$ and IL-6 are predominantly produced by TAMs in hepatocellular carcinoma (HCC), which induce EMT and activate the STAT3 pathway, respectively, to promote liver CSC properties [50,51]. Milk-fat globule EGF-8 (MFG-E8), a growth factor identified to involve in phagocytosis and immune suppression $[52,53]$, is secreted by TAMs to activate STAT3 and Hedgehog pathways that trigger tumorigenicity and drug resistance in CSCs from various cancers [54]. It is clear that the interplay between CSCs and TAMs coordinately regulates tumor progression.

2.6. Myeloid-Derived Suppressor Cells. MDSCs are a heterogeneous population of myeloid-originated progenitor cells. In mice, these cells are characterized as $\mathrm{CD}_{11 b^{+}} \mathrm{Grl}^{+}$, whereas in humans, their phenotype is $\mathrm{Lin}^{-} \mathrm{HLA}^{-} \mathrm{DR}^{-} \mathrm{CD}_{3} 3^{+}$or $\mathrm{CD}_{11}{ }^{+} \mathrm{CD} 14^{-} \mathrm{CD} 33^{+}$[55-58]. As the name indicates, the main feature of MDSCs is their function on immunosuppression. MDSCs suppress immune function primarily through multiple mechanisms, including the production of arginase, inducible nitric oxide synthase (iNOS), reactive oxygen species (ROS), cyclooxygenase-2 (COX-2), and TGF$\beta$, which together inhibit the proliferation and function of $\mathrm{T}$ cells $[59,60]$. Recent studies have demonstrated that MDSCs are actively recruited into tumors and these tumor-associated MDSCs play an important role in tumor progression. The recruitment of MDSCs into tumor sites is primarily mediated by various cancer cells that produce chemokines, including CCL2, CCL15, CXCL5, and CXCL12 [61-64]. MDSCs are implicated in multiple stages of tumor progression, particularly the regulation of CSCs. In ovarian cancer, coculture with MDSCs stimulates the expression of miR-101 in cancer cells, which regulates CtBP2 to control the expression of stemness genes, such as NANOG, OCT-4, and SOX-2 [65].
In syngeneic mammary tumor models, CSCs displayed the elevated production of granulocyte colony-stimulating factor (G-CSF), which stimulates the recruitment of MDSCs into the tumor microenvironment. MDSCs reciprocally enhance CSC properties through the activation of Notch signaling [66]. Furthermore, tumor-infiltrated MDSCs, which showed the activation of STAT3 signaling, can enhance the stemness of pancreatic cancer cells through the induction of EMT, with a concomitant increase in the expression of stemness genes, including Snail, Slug, ZEB1, NANOG, and OCT-4 [67].

2.7. T-Regulatory Cells. The fine cross talk between CSCs and immunosuppressive cells also involves Treg cells. Treg cells are defined by the $\mathrm{CD} 4^{+} \mathrm{CD} 25^{+} \mathrm{FOXP}^{+} \mathrm{T}$ cell subpopulation, with FOXP3 as an important transcriptional regulator of Treg cell development and function [68]. Treg cell-mediated immunosuppression primarily occurs through the production of various cytokines, such as IL-10, IL-35, and TGF- $\beta$, direct cell-cell contact via gap junctions, or metabolic disruption in which CD39 and CD73, expressed on Treg cells, facilitate the conversion of ATP to adenosine, which suppresses cytotoxic T cell and/or NK cell activity [69-71]. In tumors, Treg cells are accumulated by various mechanisms, primarily involving chemokine attractions. For example, the chemokines CCL22 and CCL28 are produced by tumor cells to attract CCR4- and CCR10-expressing Treg cells, respectively, leading to the accumulation of Treg cells in various human cancers [72-74]. Indeed, the number of Treg cells inside the tumor microenvironment is associated with clinical outcome. The higher number of Treg cells within the tumor is correlated with poor prognosis in a wide array of cancers, including gastric, esophageal, pancreatic, liver, and breast cancers [75-78]. In addition to its immune-suppressive role, the functional importance of tumor-infiltrating Treg cells in regulating CSCs is starting to emerge. A recent report demonstrated that, under hypoxia, FOXP3 ${ }^{+}$Treg cells are induced to express IL-17, which drives the expansion of CSCs through the activation of Akt and MAPK signaling pathways in colorectal cancer, evidenced by the increase in the expression of colorectal CSC markers, including CD133, CD44s, and EpCAM [79]. Furthermore, Treg cells produce and secrete prostaglandin (PGE2) for immunosuppression, and PGE2 has been implicated in the regulation of CSC properties in colorectal cancer through NF- $\kappa \mathrm{B}[80,81]$.

2.8. Natural Killer Cells. The ability of natural killer (NK) cells to kill or spare depends on their expression of activating (mostly stress-induced proteins) and inhibitory (in particular MHC class I molecules) ligands on the surface of target cells. Approximately 95\% of peripheral blood $\mathrm{NK}$ cells are $\mathrm{CD} 56^{\mathrm{dim}} \mathrm{CD} 16^{+}$which exerts strong cytotoxic activity. The remaining $5 \%$ of peripheral blood NK cells are $\mathrm{CD} 56^{\text {bright }} \mathrm{CD} 16^{-}$and show cytotoxicity through strong cytokine production. $\mathrm{CD} 133^{+}$glioblastoma stem cells that are able to express high levels of the activating DNAM-1 ligands PVR and Nectin-2 and low levels of MHC class I molecules have been reported to be poorly recognized and lysed by NK cells [82]. Their cytotoxic activity was revamped following IL-2 
or IL-15 activation [82]. Breast cancer CSCs have also been reported to fail to express detectable levels of NK ligands, which is consistent with metastatic spread [83]. In melanoma and GBM, CSCs are highly resistant to NK cells and become susceptible to NK cytotoxicity only following stimulation with IL-2 [82]. However, the preferential resistance of CSC to NK cells is not the rule, as colon CSCs express lower MHC class I and higher levels of NK-activating ligands, including NKp30L and Nkp44L as compared to differentiated cells, which are responsible for the CSC susceptibility to NK cell killing [84]. Another mechanism by which cancer cells may evade from the cytotoxic effect of NK cells is the induction of apoptosis in microenvironmental immune cells through the interaction of CD95 (Apo1/Fas) with its ligand (CD95L). Interestingly, CD95R/L regulates CSC plasticity and its blockade reduces CSC in different tumor cell models, while activation of CD95R/L increases CSC number and is responsible for CSC reduced sensitivity to CD95-mediated apoptosis [85]. Collectively, CSCs are more refractory to the cytotoxic effect of NK cells in a variety of cancer types.

2.9. Other Stromal Cells. There is increasing evidence that mast cells (MCs) and their mediators are involved in the remodeling of the tumor microenvironment. Recent evidence has showed that MC regulates stemness of thyroid cancer through IL-8-Akt-Slug pathway [86]. In prostate cancer, MC increased stem/progenitor cell population via altering LncRNA-HOTAIR/PRC2-androgen receptor- (AR-) MMP9 signals [87]. In addition, neutrophils were found to play a crucial role in regulation of CSC populations. Wculek and Malanchi reported that neutrophils induced expansion of breast CSC population marked by $\mathrm{CD} 24^{+} \mathrm{CD} 90^{+}$, leading to induction of tumor initiation and lung metastasis [88].

\section{Hypoxia}

Hypoxic microenvironments in tumors result from the rapid growth of cancer cells, which exceeds the limit of blood supply [89]. In response to the hypoxia, the hypoxia-related gene expression is driven through the activated hypoxiainducible factor (HIF) and transcription factors HIF- $1 \alpha$ and HIF- $2 \alpha$ that bind to the hypoxia-regulated element (HRE) gene promoters [90-92]. The capacity of HIFs to promote cancer cell stemness has been well documented. Studies have shown that HIFs can increase the expression of stem cell markers in breast cancer [93]. Bae et al. demonstrated that hypoxia can elevate the expression of the stem cell marker SOX2 in prostate cancer cell lines [94]. In addition, the overexpression of HIF-1 $\alpha$ has been associated with stem cell marker CD44 in bladder cancer [95]. In addition to HIFs, the hypoxia-mediated overexpression of extracellular carbonic anhydrases, CAIV and CAXII, facilitates cancer cell survival and the maintenance of CSC function [96].

Given that CSC is related to metastasis and cancer cell invasion, the contribution of hypoxia to the enhanced CSC migration has been reported in several studies. The upregulation of EMT-related gene expression under hypoxic stress can enhance the invasiveness and the stem-like properties of cancer [89]. Maeda et al. showed that HIF-1 $\alpha$ is correlated with the EMT and cell migration in $\mathrm{CD}_{133^{+}}$pancreatic CSCs [97]. In addition to cancer cell invasion, hypoxia contributes to drug resistance by maintaining CSCs in a quiescent state to confer resistance to chemotherapeutics that commonly target actively dividing cancer cells [91]. Studies have reported that hypoxia promotes SOX-2-mediated drug resistance in ovarian CSCs via Notch signaling [98]. The downregulation of HIF- $1 \alpha$ using a lentivirus-mediated approach can increase the chemosensitivity in triple negative breast cancer [99]. These data demonstrated that hypoxia plays an important role in the CSC niche and is substantially involved in the regulation of cancer cell stemness.

\section{Extracellular Matrix}

The extracellular matrix (ECM) is a collection of biochemical molecules, including proteins, glycoproteins, proteoglycans, and polysaccharides, which compose the basement membrane and interstitial matrix. In normal tissue, ECM is tightly regulated during development and primarily accomplished by controlling the expression or activities of ECM enzymes at the transcriptional and translational posttranslational levels [100]. Abnormal ECM dynamics are a hallmark of cancer. For instance, various collagens, including collagen I, collagen II, collagen III, collagen V, and collagen IX, show increased deposition in the process of tumor formation [101]. In addition, many other ECM components and their receptors such as heparan sulfate proteoglycans and CD44 are frequently overexpressed in cancer [102, 103]. As one of the major parts of the CSC niche, ECM provides both structural and biochemical support to the CSC and plays a critical role in cancer progression. ECM receptors enable the CSC to anchor in the niche where the stem cell properties could be maintained [104]. In addition, the ECM binds to various growth factors that interact with CSCs to maintain stem cells in a proliferative state. For example, in glioblastoma, the growth of glioblastoma stem cells can be enhanced by ECM protein laminin- $\alpha 2$ [105]. Versican G3, which is overexpressed in breast carcinoma, can inhibit cell differentiation and promote self-renewal, thereby increasing CSC properties [106, 107]. Matrix metalloproteinases that degrade and modify the ECM are upregulated in breast cancer, facilitating the EMT process [108]. Hyaluronan interacts with the cell surface protein CD44, enhancing CSC properties by activating the stem cell marker NANOG [109]. In addition to the stem cell properties, HA-CD44 interactions can also stimulate the overexpression of proteins for multidrug resistance in cancer and CSC [110]. The changes in ECM dynamics may contribute to the disruption of asymmetric stem cell division, leading to CSC overexpansion [111]. When compared with normal tissues, malignant tumors typically are characterized as stiffer due to contraction of collagen in the extracellular matrix by malignant and stromal cells [112]. On single tumor cell level, tumor stiffness was measured by atomic force microscopy mechanical measurement [113]. For in vivo measurement of tumor stiffness, compression and indentation tests were performed on fresh tumor tissues and orthotopic tumors and subcutaneous tumors derived from multiple HCC cell lines [114]. Matrix stiffness in ECM also played crucial role 
TABLE 1: Strategies of targeting the CSC niches for cancer treatment and their respective development status.

\begin{tabular}{|c|c|c|c|}
\hline Inhibitors/antibodies & Molecular targets & $\begin{array}{c}\text { Phases of } \\
\text { development }\end{array}$ & References \\
\hline \multicolumn{4}{|c|}{ CAFs } \\
\hline PT630 (FAP inhibitor) & FAP- $\alpha$ & Preclinical & {$[5]$} \\
\hline NK4 (anti-HGF monoclonal antibody) & HGF/MET & Preclinical & {$[6]$} \\
\hline AMG337 (MET kinase inhibitor) & MET & Preclinical & [7] \\
\hline Rebimastat (MMP inhibitor) & MMPs & Phase II clinical trial & NCT00040755 \\
\hline AMD3100 (CXCR4 antagonist) & SDF-1/CXCR4 & Preclinical & {$[8]$} \\
\hline GC1008 (anti-TGF- $\beta$ monoclonal antibody) & TGF- $\beta$ & Phase II clinical trial & NCT01401062 \\
\hline \multicolumn{4}{|c|}{ Tumor vasculatures } \\
\hline Sorafenib (tyrosine kinase inhibitor) & VEGFRs, PDGFRs, KIT & FDA-approved & NDA021923 \\
\hline Sunitinib (tyrosine kinase inhibitor) & VEGFRs, PDGFRs, KIT & FDA-approved & NDA021938 \\
\hline MK0752 ( $\gamma$-secretase inhibitor) & $\gamma$-secretase & Phase I clinical trial & NCT00106145 \\
\hline OMP21M18 (anti-DLL4 monoclonal antibody) & DLL4 & Phase I clinical trial & NCT01189968 \\
\hline OMP52M51 (anti-Notch1 monoclonal antibody) & Notchl & Phase I clinical trial & NCT01778439 \\
\hline \multicolumn{4}{|l|}{ ( } \\
\hline PLX3397 (CSF-1R inhibitor) & CSF-1R & Phase I/II clinical trial & NCT01596751 \\
\hline AMG820 (anti-CSF-1R monoclonal antibody) & CSF-1R & Phase I/II clinical trial & NCT02713529 \\
\hline \multirow{2}{*}{ Zoledronate, clodronate, ibandronate } & \multirow{2}{*}{ Deplete macrophages } & \multirow{2}{*}{ Phase III clinical trial } & NCT00127205 \\
\hline & & & NCT00009945 \\
\hline 852A (TLR7 agonist) & TLR7 & Phase II clinical trial & NCT00319748 \\
\hline \multirow{2}{*}{ Imiquimod (TLR7 agonist) } & \multirow{2}{*}{ TLR7 } & \multirow{2}{*}{ Phase II clinical trial } & NCT00899574 \\
\hline & & & NCT00821964 \\
\hline \multirow{3}{*}{ Tadalafil (PDE-5 inhibitors) } & MDSCs & & \\
\hline & \multirow{2}{*}{ PDE-5 } & Pilot study & NCT00843635 \\
\hline & & Phase II clinical trial & NCT00752115 \\
\hline NCX4016 (Nitric oxide-releasing aspirin derivative) & iNOS and arginase & $\begin{array}{l}\text { Phase I clinical trial } \\
\text { (Prevention purpose) }\end{array}$ & NCT00331786 \\
\hline L-NAME (arginase inhibitor) & Arginase & Preclinical & {$[9]$} \\
\hline \multirow[t]{2}{*}{ All-trans retinoic acid } & Inducing MDSC differentiation & Phase II clinical trial & NCT00617409 \\
\hline & \multicolumn{3}{|l|}{ Treg cells } \\
\hline MEDI6383 (OX40 agonist) & OX40 & Phase I clinical trial & NCT02221960 \\
\hline Ipilimumab (anti-CTLA4 monoclonal antibody) & CTLA4 & FDA-approved & BLA125377 \\
\hline \multicolumn{4}{|c|}{ Hypoxia } \\
\hline TH-302 (hypoxia-activated prodrug) & Hypoxia & Phase III clinical trial & NCT01746979 \\
\hline AQ4N (hypoxia-activated prodrug) & Hypoxia & Phase I/II clinical trial & NCT00394628 \\
\hline \multirow{3}{*}{ PEGPH20 (recombinant hyaluronidase) } & $E C M$ & & \\
\hline & \multirow{2}{*}{ Hyaluronan } & Phase II clinical trial & NCT01839487 \\
\hline & & Phase III clinical trial & NCT02715804 \\
\hline
\end{tabular}

in regulation of CSC plasticity. Tan et al. demonstrated that melanoma CSCs exhibited plasticity in mechanical stiffening, histone 3 lysine residue 9 (H3K9) methylation, Sox2 expression, and self-renewal. Three-dimensional (3D) soft fibrin matrices promote $\mathrm{H} 3 \mathrm{~K} 9$ demethylation and increase Sox2 expression and self-renewal, whereas stiff ones exert opposite effects [115]. More recently, it was found that breast CSC markers are activated synergistically in response to stiff, hypoxic conditions and that ILK is an essential regulator of breast CSCs [116]. The effect of matrix stiffness on CSC marker expression depends on cancer cell's tissue origin [117].

\section{Conclusions}

Mounting evidence suggests that CSCs are the root of cancers and are responsible for metastasis, resistance to conventional therapies, and tumor relapse. The state and survival of CSCs are controlled by various extrinsic factors derived from the microenvironment where the cells reside. As CSCs have to be 
eradicated to prevent disease relapse or metastasis, targeting the niche factors that regulate CSCs represents an attractive therapeutic strategy for cancer treatment. Considering the encouraging results of several preclinical studies for such therapeutic approaches, targeting the CSC niche is clinically feasible [118] (Table 1). A better understanding of CSC biology and the cross talk with its niche might enable the identification of potential therapeutic targets for the development of more effective anticancer treatments.

\section{Abbreviations}

CAFs: Cancer-associated fibroblasts

CSCs: Cancer stem cells

CTSB: Cathepsin B

COX-2: Cyclooxygenase-2

EGF: Epidermal growth factor

EMT: Epithelial-to-mesenchymal transition

ECM: Extracellular matrix

bFGF: fibroblast growth factor

G-CSF: Granulocyte colony-stimulating factor

HGF: Hepatocyte growth factor

HIF: Hypoxia-inducible factor

HRE: Hypoxia-regulated element

iNOS: Inducible nitric oxide synthase

IGF: Insulin-like growth factor

IL: Interleukin

MCs: Mast cells

MDSC: Myeloid-derived suppressor cell

NK: $\quad$ Natural killer

PDGF: Platelet-derived growth factor

PD-L1: Programmed death-ligand 1

PGE2: Prostaglandin

ROS: Reactive oxygen species

SDF-1: Stromal-derived factor 1

TGF- $\beta$ : Transforming growth factor beta

VEGF: Vascular endothelial growth factor.

\section{Competing Interests}

The authors declare that they have no competing interests.

\section{Acknowledgments}

The study was supported by the Health and Medical Research Fund (03142736) and Hong Kong Research Grants Council General Research Fund (PolyU 17111614 and 17120215).

\section{References}

[1] L. V. Nguyen, R. Vanner, P. Dirks, and C. J. Eaves, "Cancer stem cells: an evolving concept," Nature Reviews Cancer, vol. 12, no. 2, pp. 133-143, 2012.

[2] A. Borah, S. Raveendran, A. Rochani, T. Maekawa, and D. S. Kumar, "Targeting self-renewal pathways in cancer stem cells: clinical implications for cancer therapy," Oncogenesis, vol. 4, no. 11, article no. e177, 2015.

[3] J. Kim and S. H. Orkin, "Embryonic stem cell-specific signatures in cancer: insights into genomic regulatory networks and implications for medicine," Genome Medicine, vol. 3, no. 11, article 75, 2011.

[4] M. C. Cabrera, R. E. Hollingsworth, and E. M. Hurt, "Cancer stem cell plasticity and tumor hierarchy," World Journal of Stem Cells, vol. 7, no. 1, pp. 27-36, 2015.

[5] A. M. Santos, J. Jung, N. Aziz, J. L. Kissil, and E. Puré, “Targeting fibroblast activation protein inhibits tumor stromagenesis and growth in mice," Journal of Clinical Investigation, vol. 119, no. 12, pp. 3613-3625, 2009.

[6] S. Mizuno and T. Nakamura, "HGF-MET cascade, a key target for inhibiting cancer metastasis: the impact of NK4 discovery on cancer biology and therapeutics," International Journal of Molecular Sciences, vol. 14, no. 1, pp. 888-919, 2013.

[7] Z. Du, S. Caenepeel, Y. Shen et al., "Preclinical evaluation of AMG 337, a highly selective small molecule MET inhibitor, in Hepatocellular Carcinoma," Molecular Cancer Therapeutics, vol. 15, no. 6, pp. 1227-1237, 2016.

[8] C. Feig, J. O. Jones, M. Kraman et al., "Targeting CXCL12 from FAP-expressing carcinoma-associated fibroblasts synergizes with anti-PD-L1 immunotherapy in pancreatic cancer," Proceedings of the National Academy of Sciences of the United States of America, vol. 110, no. 50, pp. 20212-20217, 2013.

[9] G. Capuano, N. Rigamonti, M. Grioni, M. Freschi, and M. Bellone, "Modulators of arginine metabolism support cancer immunosurveillance," BMC Immunology, vol. 10, article no. 1, 2009.

[10] P. Cirri and P. Chiarugi, "Cancer associated fibroblasts: the dark side of the coin," American Journal of Cancer Research, vol. 1, no. 4, pp. 482-497, 2011.

[11] R. Kalluri and M. Zeisberg, "Fibroblasts in cancer," Nature Reviews Cancer, vol. 6, no. 5, pp. 392-401, 2006.

[12] K. Forsberg, I. Valyi-Nagy, C.-H. Heldin, M. Herlyn, and B. Westermark, "Platelet-derived growth factor (PDGF) in oncogenesis: development of a vascular connective tissue stroma in xenotransplanted human melanoma producing PDGF-BB," Proceedings of the National Academy of Sciences of the United States of America, vol. 90, no. 2, pp. 393-397, 1993.

[13] E. Giannoni, F. Bianchini, L. Masieri et al., "Reciprocal activation of prostate cancer cells and cancer-associated fibroblasts stimulates epithelial-mesenchymal transition and cancer stemness," Cancer Research, vol. 70, no. 17, pp. 6945-6956, 2010.

[14] L. J. A. C. Hawinkels, M. Paauwe, H. W. Verspaget et al., "Interaction with colon cancer cells hyperactivates TGF- $\beta$ signaling in cancer-associated fibroblasts," Oncogene, vol. 33, no. 1, pp. 97-107, 2014.

[15] F. Strutz, M. Zeisberg, B. Hemmerlein et al., "Basic fibroblast growth factor expression is increased in human renal fibrogenesis and may mediate autocrine fibroblast proliferation," Kidney International, vol. 57, no. 4, pp. 1521-1538, 2000.

[16] L. Vermeulen, F. De Sousa E Melo, M. van der Heijden et al., "Wnt activity defines colon cancer stem cells and is regulated by the microenvironment," Nature Cell Biology, vol. 12, no. 5, pp. 468-476, 2010.

[17] E. Y. T. Lau, J. Lo, B. Y. L. Cheng et al., "Cancer-associated fibroblasts regulate tumor-initiating cell plasticity in hepatocellular carcinoma through c-Met/FRA1/HEY1 signaling," Cell Reports, vol. 15, no. 6, pp. 1175-1189, 2016.

[18] W.-J. Chen, C.-C. Ho, Y.-L. Chang et al., "Cancer-associated fibroblasts regulate the plasticity of lung cancer stemness via paracrine signalling," Nature Communications, vol. 5, article 3472, 2014. 
[19] W. L. Tam, H. Lu, J. Buikhuisen et al., "Protein kinase $\mathrm{C} \alpha$ is a central signaling node and therapeutic target for breast cancer stem cells," Cancer Cell, vol. 24, no. 3, pp. 347-364, 2013.

[20] U. Wellner, J. Schubert, U. C. Burk et al., "The EMT-activator ZEB1 promotes tumorigenicity by repressing stemnessinhibiting microRNAs," Nature Cell Biology, vol. 11, no. 12, pp. 1487-1495, 2009.

[21] P. S. H. Soon, E. Kim, C. K. Pon et al., "Breast cancer-associated fibroblasts induce epithelial-to-mesenchymal transition in breast cancer cells," Endocrine-Related Cancer, vol. 20, no. 1, pp. 1-12, 2013.

[22] Y. Yu, C.-H. Xiao, L.-D. Tan, Q.-S. Wang, X.-Q. Li, and Y.-M. Feng, "Cancer-associated fibroblasts induce epithelialmesenchymal transition of breast cancer cells through paracrine TGF- $\beta$ signalling," British Journal of Cancer, vol. 110, no. 3, pp. 724-732, 2014.

[23] G. De Pergola and F. Silvestris, "Obesity as a major risk factor for cancer," Journal of Obesity, vol. 2013, Article ID 291546, 11 pages, 2013.

[24] G. Fantuzzi, "Adipose tissue, adipokines, and inflammation," Journal of Allergy and Clinical Immunology, vol. 115, no. 5, pp. 911-920, 2005.

[25] D. E. Feldman, C. Chen, V. Punj, H. Tsukamoto, and K. Machida, "Pluripotency factor-mediated expression of the leptin receptor (OB-R) links obesity to oncogenesis through tumor-initiating stem cells," Proceedings of the National Academy of Sciences of the United States of America, vol. 109, no. 3, pp. 829-834, 2012.

[26] M. Picon-Ruiz, C. Pan, K. Drews-Elger et al., "Interactions between adipocytes and breast cancer cells stimulate cytokine production and drive Src/Sox2/miR-302b-mediated malignant progression," Cancer Research, vol. 76, no. 2, pp. 491-504, 2016.

[27] K.-D. Tang, J. Liu, L. Jovanovic et al., "Adipocytes promote prostate cancer stem cell self-renewal through amplification of the cholecystokinin autocrine loop," Oncotarget, vol. 7, no. 4, pp. 4939-4948, 2016.

[28] S. Kumar, D. Kumar, K. Raina, R. Agarwal, and C. Agarwal, "Functional modification of adipocytes by grape seed extract impairs their pro-tumorigenic signaling on colon cancer stem cells and the daughter cancer cells," Oncotarget, vol. 5, no. 20, pp. 10151-10169, 2014.

[29] S. Bao, Q. Wu, S. Sathornsumetee et al., "Stem cell-like glioma cells promote tumor angiogenesis through vascular endothelial growth factor," Cancer Research, vol. 66, no. 16, pp. 7843-7848, 2006.

[30] E. Monzani, F. Facchetti, E. Galmozzi et al., "Melanoma contains CD133 and ABCG2 positive cells with enhanced tumourigenic potential," European Journal of Cancer, vol. 43, no. 5, pp. 935946, 2007.

[31] X.-R. Yang, Y. Xu, B. Yu et al., "High expression levels of putative hepatic stem/progenitor cell biomarkers related to tumour angiogenesis and poor prognosis of hepatocellular carcinoma," Gut, vol. 59, no. 7, pp. 953-962, 2010.

[32] L. Cheng, Z. Huang, W. Zhou et al., "Glioblastoma stem cells generate vascular pericytes to support vessel function and tumor growth," Cell, vol. 153, no. 1, pp. 139-152, 2013.

[33] L. Ricci-Vitiani, R. Pallini, M. Biffoni et al., "Tumour vascularization via endothelial differentiation of glioblastoma stem-like cells," Nature, vol. 468, no. 7325, pp. 824-830, 2010.

[34] T. S. Zhu, M. A. Costello, C. E. Talsma et al., "Endothelial cells create a stem cell niche in glioblastoma by providing $\mathrm{NOTCH}$ ligands that nurture self-renewal of cancer stem-like cells," Cancer Research, vol. 71, no. 18, pp. 6061-6072, 2011.

[35] N. Charles, T. Ozawa, M. Squatrito et al., "Perivascular nitric oxide activates notch signaling and promotes stem-like character in PDGF-induced glioma cells," Cell Stem Cell, vol. 6, no. 2, pp. 141-152, 2010.

[36] J. Lu, X. Ye, F. Fan et al., "Endothelial cells promote the colorectal cancer stem cell phenotype through a soluble form of Jagged-1," Cancer Cell, vol. 23, no. 2, pp. 171-185, 2013.

[37] Z. Zhang, Z. Dong, I. S. Lauxen, M. S. Filho, and J. E. Nör, "Endothelial cell-secreted EGF induces epithelial to mesenchymal transition and endows head and neck cancer cells with stem-like phenotype," Cancer Research, vol. 74, no. 10, pp. 28692881, 2014.

[38] S. L. Topalian, J. M. Taube, R. A. Anders, and D. M. Pardoll, "Mechanism-driven biomarkers to guide immune checkpoint blockade in cancer therapy," Nature Reviews Cancer, vol. 16, no. 5, pp. 275-287, 2016.

[39] W. Zou, "Immunosuppressive networks in the tumour environment and their therapeutic relevance," Nature Reviews Cancer, vol. 5, no. 4, pp. 263-274, 2005.

[40] F. Dituri, A. Mazzocca, G. Giannelli, and S. Antonaci, "PI3K functions in cancer progression, anticancer immunity and immune evasion by tumors," Clinical and Developmental Immunology, vol. 2011, Article ID 947858, 10 pages, 2011.

[41] T. Di Tomaso, S. Mazzoleni, E. Wang et al., "Immunobiological characterization of cancer stem cells isolated from glioblastoma patients," Clinical Cancer Research, vol. 16, no. 3, pp. 800-813, 2010.

[42] Y. Lee, J. H. Shin, M. Longmire et al., "Cd $44^{+}$cells in head and neck squamous cell carcinoma suppress t-cell-mediated immunity by selective constitutive and inducible expression of PD-L1," Clinical Cancer Research, vol. 22, no. 14, pp. 3571-3581, 2016.

[43] T. Kitamura, B.-Z. Qian, and J. W. Pollard, "Immune cell promotion of metastasis," Nature Reviews Immunology, vol. 15, no. 2, pp. 73-86, 2015.

[44] F. O. Martinez and S. Gordon, "The M1 and M2 paradigm of macrophage activation: time for reassessment," F1000Prime Reports, vol. 6, article no. 13, 2014.

[45] J. G. Quatromoni and E. Eruslanov, "Tumor-associated macrophages: function, phenotype, and link to prognosis in human lung cancer," American Journal of Translational Research, vol. 4, no. 4, pp. 376-389, 2012.

[46] A. Sica, T. Schioppa, A. Mantovani, and P. Allavena, "Tumourassociated macrophages are a distinct M2 polarised population promoting tumour progression: potential targets of anti-cancer therapy," European Journal of Cancer, vol. 42, no. 6, pp. 717-727, 2006.

[47] A. Wu, J. Wei, L.-Y. Kong et al., "Glioma cancer stem cells induce immunosuppressive macrophages/microglia," Neuro-Oncology, vol. 12, no. 11, pp. 1113-1125, 2010.

[48] H. Lu, K. R. Clauser, W. L. Tam et al., "A breast cancer stem cell niche supported by juxtacrine signalling from monocytes and macrophages," Nature Cell Biology, vol. 16, no. 11, pp. 1105-1117, 2014.

[49] J. Yang, D. Liao, C. Chen et al., "Tumor-associated macrophages regulate murine breast cancer stem cells through a novel paracrine EGFR/Stat3/Sox-2 signaling pathway," STEM CELLS, vol. 31, no. 2, pp. 248-258, 2013. 
[50] Q.-M. Fan, Y.-Y. Jing, G.-F. Yu et al., “Tumor-associated macrophages promote cancer stem cell-like properties via transforming growth factor-betal-induced epithelialmesenchymal transition in hepatocellular carcinoma," Cancer Letters, vol. 352, no. 2, pp. 160-168, 2014.

[51] S. Wan, E. Zhao, I. Kryczek et al., "Tumor-associated macrophages produce interleukin 6 and signal via STAT3 to promote expansion of human hepatocellular carcinoma stem cells," Gastroenterology, vol. 147, no. 6, pp. 1393-1404, 2014.

[52] R. Hanayama, M. Tanaka, K. Miwa, A. Shinohara, A. Iwamatsu, and S. Nagata, "Identification of a factor that links apoptotic cells to phagocytes," Nature, vol. 417, no. 6885, pp. 182-187, 2002.

[53] M. Jinushi, M. Sato, A. Kanamoto et al., "Milk fat globule epidermal growth factor-8 blockade triggers tumor destruction through coordinated cell-autonomous and immune-mediated mechanisms," Journal of Experimental Medicine, vol. 206, no. 6, pp. 1317-1326, 2009.

[54] M. Jinushi, S. Chiba, H. Yoshiyama et al., "Tumor-associated macrophages regulate tumorigenicity and anticancer drug responses of cancer stem/initiating cells," Proceedings of the National Academy of Sciences of the United States of America, vol. 108, no. 30, pp. 12425-12430, 2011.

[55] S. Kusmartsev, Y. Nefedova, D. Yoder, and D. I. Gabrilovich, "Antigen-specific inhibition of CD8+ T cell response by immature myeloid cells in cancer is mediated by reactive oxygen species," Journal of Immunology, vol. 172, no. 2, pp. 989-999, 2004.

[56] S. Nagaraj and D. I. Gabrilovich, "Myeloid-derived suppressor cells in human cancer," Cancer Journal, vol. 16, no. 4, pp. 348353, 2010.

[57] I. Poschke, Y. Mao, L. Adamson, F. Salazar-Onfray, G. Masucci, and R. Kiessling, "Myeloid-derived suppressor cells impair the quality of dendritic cell vaccines," Cancer Immunology, Immunotherapy, vol. 61, no. 6, pp. 827-838, 2012.

[58] J. E. Talmadge and D. I. Gabrilovich, "History of myeloidderived suppressor cells," Nature Reviews Cancer, vol. 13, no. 10, pp. 739-752, 2013.

[59] D. I. Gabrilovich and S. Nagaraj, "Myeloid-derived suppressor cells as regulators of the immune system," Nature Reviews Immunology, vol. 9, no. 3, pp. 162-174, 2009.

[60] S. Ostrand-Rosenberg, "Myeloid-derived suppressor cells: more mechanisms for inhibiting antitumor immunity," Cancer Immunology, Immunotherapy, vol. 59, no. 10, pp. 1593-1600, 2010.

[61] B. Huang, Z. Lei, J. Zhao et al., "CCL2/CCR2 pathway mediates recruitment of myeloid suppressor cells to cancers," Cancer Letters, vol. 252, no. 1, pp. 86-92, 2007.

[62] S. Inamoto, Y. Itatani, T. Yamamoto et al., "Loss of SMAD4 promotes colorectal cancer progression by accumulation of myeloid-derived suppressor cells through the CCL15-CCR1 chemokine axis," Clinical Cancer Research, vol. 22, no. 2, pp. 492-501, 2016.

[63] L.-Y. OuYang, X.-J. Wu, S.-B. Ye et al., "Tumor-induced myeloid-derived suppressor cells promote tumor progression through oxidative metabolism in human colorectal cancer," Journal of Translational Medicine, vol. 13, no. 1, article 47, 2015.

[64] L. Yang, J. Huang, X. Ren et al., "Abrogation of TGF $\beta$ signaling in mammary carcinomas recruits Gr-1+CD11b+ myeloid cells that promote metastasis," Cancer Cell, vol. 13, no. 1, pp. 23-35, 2008.

[65] T. X. Cui, I. Kryczek, L. Zhao et al., "Myeloid-derived suppressor cells enhance stemness of cancer cells by inducing
microRNA101 and suppressing the corepressor CTBP2," Immunity, vol. 39, no. 3, pp. 611-621, 2013.

[66] T. Welte, I. S. Kim, L. Tian et al., "Oncogenic mTOR signalling recruits myeloid-derived suppressor cells to promote tumour initiation," Nature Cell Biology, vol. 18, no. 6, pp. 632-644, 2016.

[67] R. Z. Panni, D. E. Sanford, B. A. Belt et al., "Tumor-induced STAT3 activation in monocytic myeloid-derived suppressor cells enhances stemness and mesenchymal properties in human pancreatic cancer," Cancer Immunology, Immunotherapy, vol. 63, no. 5, pp. 513-528, 2014.

[68] S. Sakaguchi, M. Miyara, C. M. Costantino, and D. A. Hafler, "FOXP3 + regulatory T cells in the human immune system," Nature Reviews Immunology, vol. 10, no. 7, pp. 490-500, 2010.

[69] T. Bopp, C. Becker, M. Klein et al., "Cyclic adenosine monophosphate is a key component of regulatory $\mathrm{T}$ cellmediated suppression," Journal of Experimental Medicine, vol. 204, no. 6, pp. 1303-1310, 2007.

[70] S. Deaglio, K. M. Dwyer, W. Gao et al., "Adenosine generation catalyzed by CD39 and CD73 expressed on regulatory $\mathrm{T}$ cells mediates immune suppression," Journal of Experimental Medicine, vol. 204, no. 6, pp. 1257-1265, 2007.

[71] D. A. A. Vignali, L. W. Collison, and C. J. Workman, "How regulatory T cells work," Nature Reviews Immunology, vol. 8, no. 7, pp. 523-532, 2008.

[72] A. Facciabene, X. Peng, I. S. Hagemann et al., "Tumour hypoxia promotes tolerance and angiogenesis via CCL28 and T(reg) cells," Nature, vol. 475, no. 7355, pp. 226-230, 2011.

[73] T. Maruyama, K. Kono, S. Izawa et al., "CCL17 and CCL22 chemokines within tumor microenvironment are related to infiltration of regulatory $\mathrm{T}$ cells in esophageal squamous cell carcinoma," Diseases of the Esophagus, vol. 23, no. 5, pp. 422429, 2010.

[74] Y. Mizukami, K. Kono, Y. Kawaguchi et al., "CCL17 and CCL22 chemokines within tumor microenvironment are related to accumulation of Foxp $3^{+}$regulatory T cells in gastric cancer," International Journal of Cancer, vol. 122, no. 10, pp. 2286-2293, 2008.

[75] G. J. Bates, S. B. Fox, C. Han et al., "Quantification of regulatory $\mathrm{T}$ cells enables the identification of high-risk breast cancer patients and those at risk of late relapse," Journal of Clinical Oncology, vol. 24, no. 34, pp. 5373-5380, 2006.

[76] N. Hiraoka, K. Onozato, T. Kosuge, and S. Hirohashi, "Prevalence of FOXP3+ regulatory $\mathrm{T}$ cells increases during the progression of pancreatic ductal adenocarcinoma and its premalignant lesions," Clinical Cancer Research, vol. 12, no. 18, pp. 5423-5434, 2006.

[77] N. Kobayashi, N. Hiraoka, W. Yamagami et al., "FOXP3 ${ }^{+}$ regulatory $\mathrm{T}$ cells affect the development and progression of hepatocarcinogenesis," Clinical Cancer Research, vol. 13, no. 3, pp. 902-911, 2007.

[78] K. Kono, H. Kawaida, A. Takahashi et al., "CD4(+)CD25high regulatory T cells increase with tumor stage in patients with gastric and esophageal cancers," Cancer Immunology, Immunotherapy, vol. 55, no. 9, pp. 1064-1071, 2006.

[79] S. Yang, B. Wang, C. Guan et al., "Foxp $3^{+} \mathrm{IL}-17^{+} \mathrm{T}$ cells promote development of cancer-initiating cells in colorectal cancer," Journal of Leukocyte Biology, vol. 89, no. 1, pp. 85-91, 2011.

[80] M. Mahic, S. Yaqub, C. C. Johansson, K. Taskén, and E. M. Aandahl, "FOXP3+CD4+CD25+ adaptive regulatory $\mathrm{T}$ cells express cyclooxygenase- 2 and suppress effector $\mathrm{T}$ cells by a prostaglandin E2-dependent mechanism," Journal of Immunology, vol. 177, no. 1, pp. 246-254, 2006. 
[81] D. Wang, L. Fu, H. Sun, L. Guo, and R. N. DuBois, "Prostaglandin $\mathrm{E}_{2}$ promotes colorectal cancer stem cell expansion and metastasis in mice," Gastroenterology, vol. 149, no. 7, pp. 18841895.e4, 2015.

[82] R. Castriconi, A. Daga, A. Dondero et al., "NK cells recognize and kill human glioblastoma cells with stem cell-like properties," Journal of Immunology, vol. 182, no. 6, pp. 3530-3539, 2009.

[83] B. Wang, Q. Wang, Z. Wang et al., "Metastatic consequences of immune escape from NK cell cytotoxicity by human breast cancer stem cells," Cancer Research, vol. 74, no. 20, pp. 57465757, 2014.

[84] R. Tallerico, M. Todaro, S. Di Franco et al., "Human NK cells selective targeting of colon cancer-initiating cells: a role for natural cytotoxicity receptors and MHC class I molecules," Journal of Immunology, vol. 190, no. 5, pp. 2381-2390, 2013.

[85] P. Ceppi, A. Hadji, F. J. Kohlhapp et al., "CD95 and CD95L promote and protect cancer stem cells," Nature Communications, vol. 5, article no. 5238, 2014.

[86] C. Visciano, F. Liotti, N. Prevete et al., "Mast cells induce epithelial-to-mesenchymal transition and stem cell features in human thyroid cancer cells through an IL-8-Akt-Slug pathway," Oncogene, vol. 34, no. 40, pp. 5175-5186, 2015.

[87] L. Li, Q. Dang, H. Xie et al., "Infiltrating mast cells enhance prostate cancer invasion via altering LncRNA-HOTAIR/PRC2androgen receptor (AR)-MMP9 signals and increased stem/progenitor cell population," Oncotarget, vol. 6, no. 16, pp. 14179-14190, 2015.

[88] S. K. Wculek and I. Malanchi, "Neutrophils support lung colonization of metastasis-initiating breast cancer cells," Nature, vol. 528, no. 7582, pp. 413-417, 2015.

[89] T. Gao, J.-Z. Li, Y. Lu et al., “The mechanism between epithelial mesenchymal transition in breast cancer and hypoxia microenvironment," Biomedicine and Pharmacotherapy, vol. 80, pp. 393405, 2016.

[90] P. J. Ratcliffe, "HIF-1 and HIF-2: working alone or together in hypoxia?" Journal of Clinical Investigation, vol. 117, no. 4, pp. 862-865, 2007.

[91] J. P. Schöning, M. Monteiro, and W. Gu, "Drug resistance and cancer stem cells: the shared but distinct roles of hypoxiainducible factors HIF1 $\alpha$ and HIF2 $\alpha$," Clinical and Experimental Pharmacology and Physiology, vol. 44, no. 2, pp. 153-161, 2017.

[92] G. L. Semenza, "Hypoxia-inducible factor 1: oxygen homeostasis and disease pathophysiology," Trends in Molecular Medicine, vol. 7, no. 8, pp. 345-350, 2001.

[93] C. Zhang, W. I. Zhi, H. Lu et al., "Hypoxia-inducible factors regulate pluripotency factor expression by ZNF217- and ALKBH5mediated modulation of RNA methylation in breast cancer cells," Oncotarget, vol. 7, no. 40, pp. 64527-64542, 2016.

[94] K. Bae, Y. Dai, J. Vieweg, and D. W. Siemann, "Hypoxia regulates SOX2 expression to promote prostate cancer cell invasion and sphere formation," American Journal of Cancer Research, vol. 6, no. 5, pp. 1078-1088, 2016.

[95] M. I. Koukourakis, C. Kakouratos, D. Kalamida et al., "Hypoxiainducible proteins HIF1 $\alpha$ and lactate dehydrogenase LDH5, key markers of anaerobic metabolism, relate with stem cell markers and poor post-radiotherapy outcome in bladder cancer," International Journal of Radiation Biology, vol. 92, no. 7, pp. 353-363, 2016.

[96] P. C. McDonald, S. C. Chafe, and S. Dedhar, "Overcoming hypoxia-mediated tumor progression: combinatorial approaches targeting $\mathrm{pH}$ regulation, angiogenesis and immune dysfunction," Frontiers in Cell and Developmental Biology, vol. 4, article no. 27, 2016.

[97] K. Maeda, Q. Ding, M. Yoshimitsu et al., "CD133 modulate HIF$1 \alpha$ expression under hypoxia in EMT phenotype pancreatic cancer stem-like cells," International Journal of Molecular Sciences, vol. 17, no. 7, article 1025, 2016.

[98] E. J. Seo, D. K. Kim, I. H. Jang et al., "Hypoxia-NOTCH1SOX2 signaling is important for maintaining cancer stem cells in ovarian cancer," Oncotarget, vol. 7, no. 34, pp. 55624-55638, 2016.

[99] S. Li, Q. Wei, Q. Li, B. Zhang, and Q. Xiao, "Down-regulating HIF-1 $\alpha$ by lentivirus-mediated shRNA for therapy of triple negative breast cancer," Cancer Biology and Therapy, vol. 16, no. 6, pp. 866-875, 2015.

[100] A. Page-McCaw, A. J. Ewald, and Z. Werb, "Matrix metalloproteinases and the regulation of tissue remodelling," Nature Reviews Molecular Cell Biology, vol. 8, no. 3, pp. 221-233, 2007.

[101] I. J. Huijbers, M. Iravani, S. Popov et al., "A role for fibrillar collagen deposition and the collagen internalization receptor endo180 in glioma invasion," PLoS ONE, vol. 5, no. 3, Article ID e9808, 2010.

[102] C. Kainz, P. Kohlberger, C. Tempfer et al., "Prognostic value of CD44 splice variants in human stage III cervical cancer," European Journal of Cancer, vol. 31, no. 10, pp. 1706-1709, 1995.

[103] N. J. Nasser, "Heparanase involvement in physiology and disease," Cellular and Molecular Life Sciences, vol. 65, no. 11, pp. 1706-1715, 2008.

[104] E. Fuchs, T. Tumbar, and G. Guasch, "Socializing with the neighbors: stem cells and their niche," Cell, vol. 116, no. 6, pp. 769-778, 2004.

[105] J. D. Lathia, M. Li, P. E. Hall et al., "Laminin alpha 2 enables glioblastoma stem cell growth," Annals of Neurology, vol. 72, no. 5, pp. 766-778, 2012.

[106] W. W. Du, L. Fang, W. Yang et al., "The role of versican G3 domain in regulating breast cancer cell motility including effects on osteoblast cell growth and differentiation in vitroevaluation towards understanding breast cancer cell bone metastasis," BMC Cancer, vol. 12, article no. 341, 2012.

[107] W. W. Du, L. Fang, X. Yang et al., "The role of versican in modulating breast cancer cell self-renewal," Molecular Cancer Research, vol. 11, no. 5, pp. 443-455, 2013.

[108] E. S. Radisky and D. C. Radisky, "Matrix metalloproteinaseinduced epithelial-mesenchymal transition in breast cancer," Journal of Mammary Gland Biology and Neoplasia, vol. 15, no. 2, pp. 201-212, 2010.

[109] L. Y. W. Bourguignon, K. Peyrollier, W. Xia, and E. Gilad, "Hyaluronan-CD44 interaction activates stem cell marker Nanog, Stat-3-mediated MDR1 gene expression, and ankyrinregulated multidrug efflux in breast and ovarian tumor cells," Journal of Biological Chemistry, vol. 283, no. 25, pp. 17635-17651, 2008.

[110] L. Y. W. Bourguignon, "Matrix hyaluronan promotes specific microRNA upregulation leading to drug resistance and tumor progression," International Journal of Molecular Sciences, vol. 17, no. 4, article 517, 2016.

[111] P. Lu, V. M. Weaver, and Z. Werb, "The extracellular matrix: a dynamic niche in cancer progression," Journal of Cell Biology, vol. 196, no. 4, pp. 395-406, 2012.

[112] D. T. Butcher, T. Alliston, and V. M. Weaver, "A tense situation: forcing tumour progression," Nature Reviews Cancer, vol. 9, no. 2, pp. 108-122, 2009. 
[113] W. Xu, R. Mezencev, B. Kim, L. Wang, J. McDonald, and T. Sulchek, "Cell stiffness is a biomarker of the metastatic potential of ovarian cancer cells," PLoS ONE, vol. 7, no. 10, Article ID e46609, 2012.

[114] C. C.-L. Wong, A. P.-W. Tse, Y.-P. Huang et al., "Lysyl oxidaselike 2 is critical to tumor microenvironment and metastatic niche formation in hepatocellular carcinoma," Hepatology, vol. 60, no. 5, pp. 1645-1658, 2014.

[115] Y. Tan, A. Tajik, J. Chen et al., "Matrix softness regulates plasticity of tumour-repopulating cells via $\mathrm{H} 3 \mathrm{~K} 9$ demethylation and Sox 2 expression," Nature Communications, vol. 5, article no. 4619, 2014.

[116] M. F. Pang, M. J. Siedlik, S. Han, M. Stallings-Mann, D. C. Radisky, and C. M. Nelson, "Tissue stiffness and hypoxia modulate the integrin-linked kinase ILK to control breast cancer stem-like cells," Cancer Research, vol. 76, no. 18, pp. 52775287, 2016.

[117] E. Jabbari, S. K. Sarvestani, L. Daneshian, and S. Moeinzadeh, "Optimum 3D matrix stiffness for maintenance of cancer stem cells is dependent on tissue origin of cancer cells," PLoS ONE, vol. 10, no. 7, Article ID e0132377, 2015.

[118] D. L. Dragu, L. G. Necula, C. Bleotu, C. C. Diaconu, and M. Chivu-Economescu, "Therapies targeting cancer stem cells: current trends and future challenges," World Journal of Stem Cells, vol. 7, no. 9, pp. 1185-1201, 2015. 

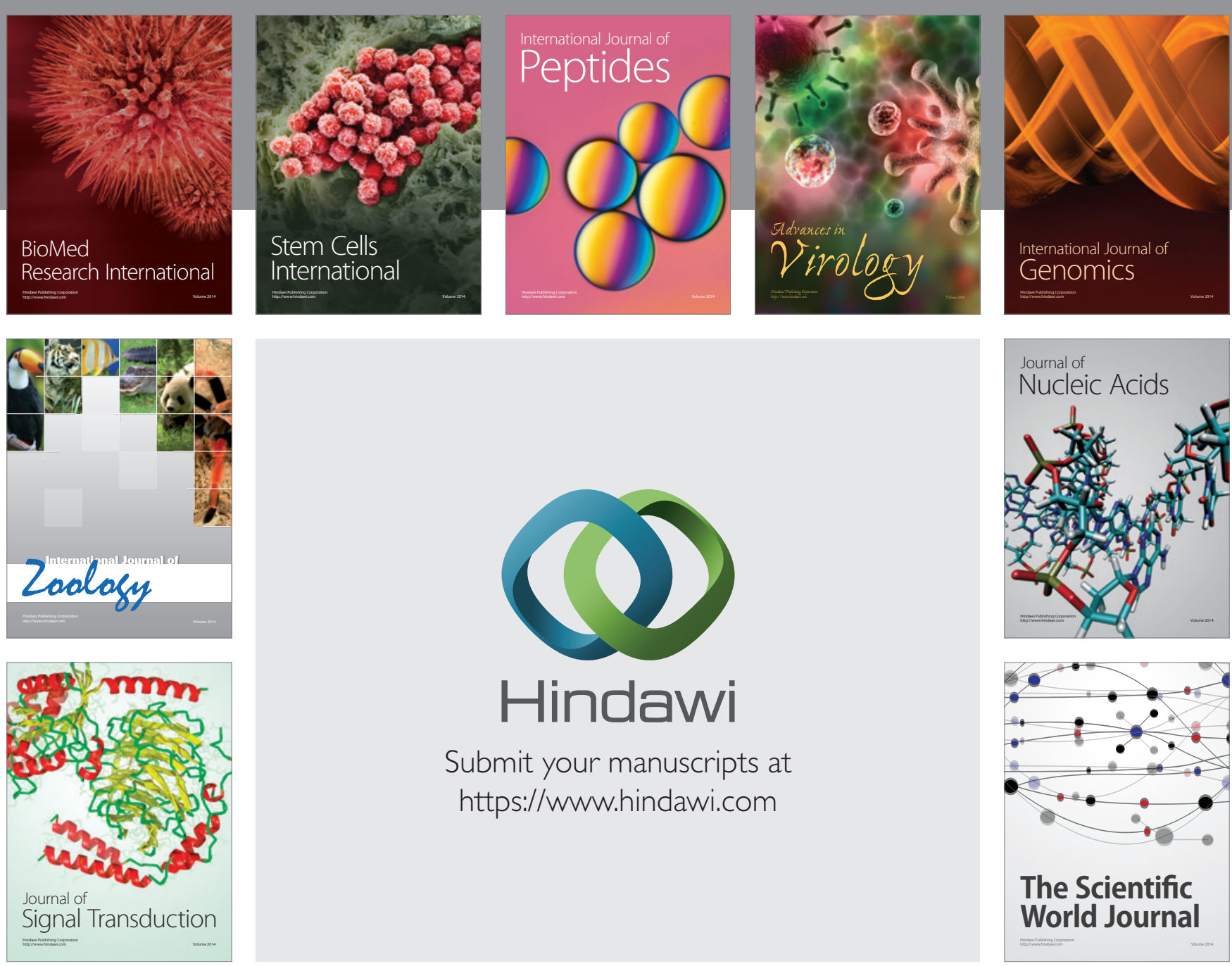

Submit your manuscripts at

https://www.hindawi.com
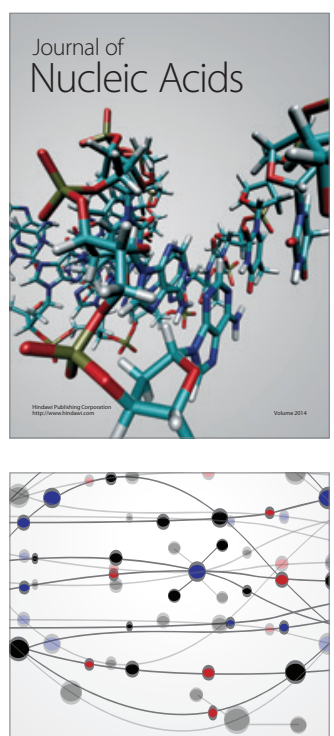

The Scientific World Journal
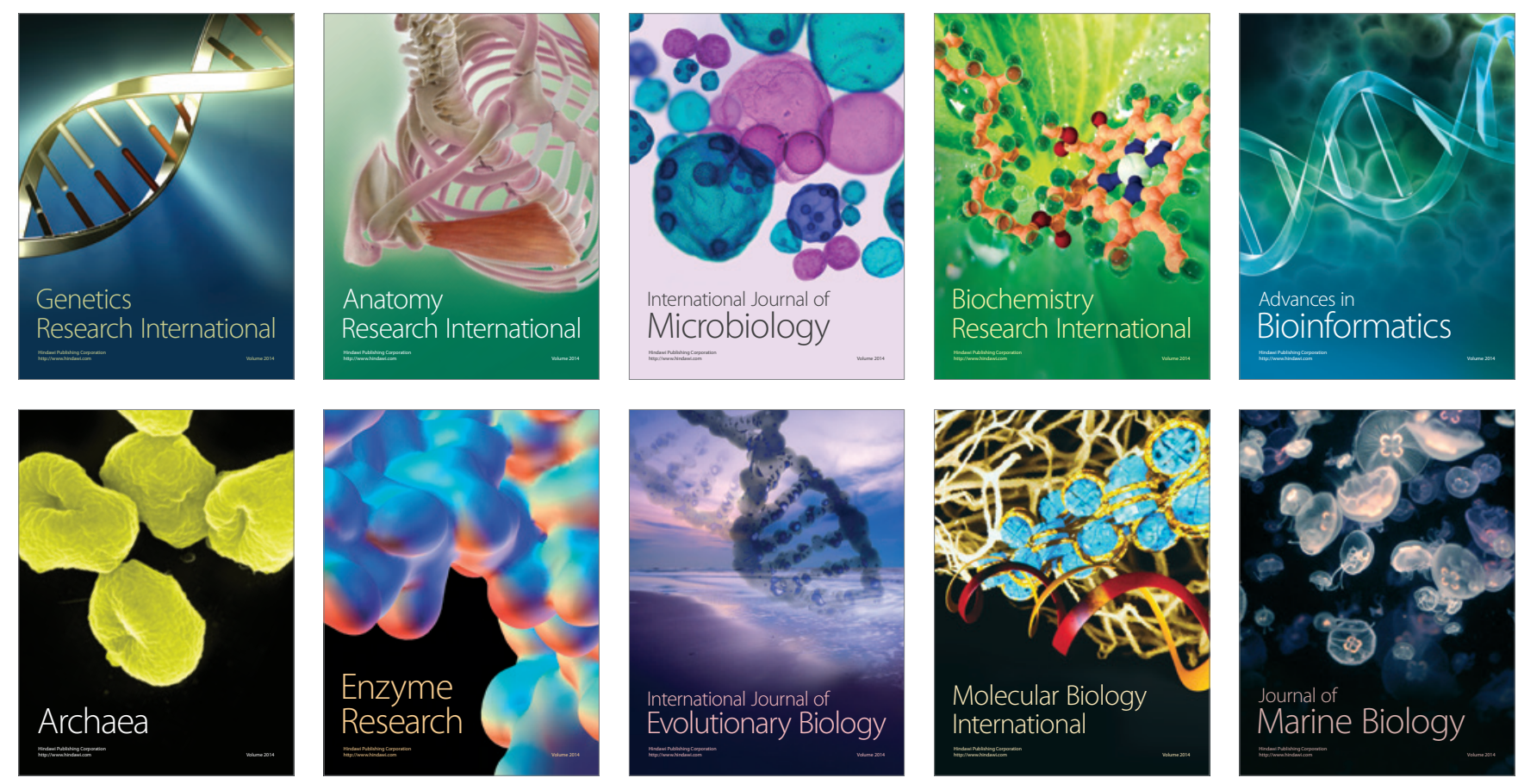